\title{
LA MOVILIDAD EN SU AMBIENTE: PRÁCTICAS Y EXPERIENCIAS DE MOVILIDAD COTIDIANA MAPUCHE-WILLICHE EN CONTEXTOS RURALES. EVIDENCIAS DESDE LA COMUNA DE PUYEHUE, REGIÓN DE LOS LAGOS, CHILE*
}

\author{
THE MOBILITY IN ITS ENVIROMENT: \\ MAPUCHE-WILLICHE DAILY MOBILITY PRACTICES AND EXPERIENCES \\ IN RURAL CONTEXTS. EVIDENCE FROM THE CITY OF PUYEHUE, \\ REGION DE LOS LAGOS, CHILE
}

\author{
Alejandra Lazo Corvalán ${ }^{* *}$, Hernán Riquelme Brevis ${ }^{* * *}$ y Viviana Huiliñir-Curío ${ }^{* * * * *}$
}

\begin{abstract}
La movilidad cotidiana mapuche-williche constituye una práctica histórica y cultural de la zona centro-sur de Chile, sin embargo, las representaciones y experiencias de movilidad rural de los habitantes de estos territorios han sido escasamente investigadas. A partir de una metodología cualitativa, en este trabajo se analiza la movilidad en su ambiente con el propósito de develar las actividades, personas, objetos y lugares que constituyen la movilidad rural e indígena en Los Lagos. Dentro de los principales hallazgos destaca una cosmología particular del territorio, relaciones sociales circulares significativas, factores rítmicos propios de la cultura mapuche y aspectos identitarios de la movilidad.

Palabras claves: Movilidad indígena, ruralidad, territorialidad, vida cotidiana.
\end{abstract}

Mapuche-Williche mobility is a historical and cultural practice of the central-south Chile area. However, the representations and experiences of rural mobility of the inhabitants of these territories have been scarcely researched. Based on a qualitative methodology, this research analyzes the mobility in its environment to reveal the activities, people, objects, and places that are part of the indigenous and rural mobility in Los Lagos. The main findings, some elements can be highlighted: a particular cosmology of the territory, significant circular social relationships, particular rhythmic variables from the Mapuche culture and identity aspects of mobility.

Key words: Indigenous mobility, rurality, territoriality, daily life.

\section{Introducción}

Este artículo se focaliza en comprender la movilidad cotidiana de los habitantes mapuche-williche de Los Lagos, por medio de su dimensión experiencial, relacional y material de una zona predominantemente rural y marcada por la presencia histórica de comunidades indígenas. En efecto, se discutirá acerca de la movilidad mapuche-williche de Puyehue como una movilidad en su ambiente, es decir, como una circularidad relacional donde convergen prácticas, experiencias, significaciones y materialidades.

Los espacios rurales y las movilidades indígenas constituyen interesantes y novedosos casos de estudios dentro de las investigaciones de movilidades contemporáneas, siendo estas movilidades en muchos sentidos distintas a las urbanas. $\mathrm{Si}$ bien la movilidad ha sido tratada dentro de los estudios rurales en términos de análisis espacial de la migración rural-urbana y de los impactos sociales y culturales de la migración en lugares

\footnotetext{
* Resultado del proyecto FONDECYT 111710128. "La movilidad en su ambiente: Etnografía de la experiencia de los conmutantes de las ciudades Osorno y Puerto Montt en la Región de Los Lagos".

** Universidad de Los Lagos, Departamento de Arquitectura, Osorno, Chile. Correo electrónico: alejandra.lazo@ulagos.cl

*** Universidad Autónoma de Chile, Instituto de Estudios Sociales y Humanísticos, Temuco, Chile.

Correo electrónico: h.riquelmebrevis@gmail.com

**** Universidad de La Frontera, Centro Internacional de Estudios de la Patagonia (CIE-Patagonia), Núcleo de Ciencias Sociales y Humanidades, Temuco, Chile. Correo electrónico viviana.huilinir@ufrontera.cl
} 
específicos (Milbourne y Kitchen, 2014), no se ha prestado una atención crítica a las dimensiones sociales, culturales, políticas y económicas de las movilidades rurales y sus implicancias en lugares particulares.

En efecto, muchos de los habitantes de las localidades rurales e indígenas se movilizan frecuentemente a las ciudades mayores por motivos de trabajo, estudios u otras actividades. Esta situación genera desplazamientos cotidianos que se realizan en modos de transporte precarios, implicando a menudo trayectos complejos (caminar varios kilómetros, andar a caballo y "hacer dedo"), al mismo tiempo que supone poner en marcha recursos, capitales y competencias, así como formas importantes de organización del tiempo y el espacio (Jouffe \& Lazo, 2010; Lazo \& Carvajal, 2017; Lazo \& Carvajal, 2018).

Además, y desde una perspectiva territorial, el alcance de estas movilidades evidencia que la diferenciación urbano-rural no es tal en muchas regiones, como la existencia de un alto nivel en el hinterland de las conmutaciones cotidianas. Así como se plantea en algunos estudios realizados en regiones de similares características como La Araucanía (Salazar, Fonck e Irarrázaval, 2017; Riquelme y Lazo, 2019), las dinámicas socioespaciales de estos asentamientos poseen una densa interacción sociocultural definida por los flujos de población rural de origen mapuche, así como de población rural no mapuche. Asimismo, la movilidad -desde la ruralidad- debe apuntar a visibilizar las experiencias propias de los lugares en directa relación con el paisaje y los grupos humanos que allí habitan (Salazar et al., 2017), como también a la articulación de territorios familiares en el espacio (Castelleti, Villarroel, Almendares y Mercado, 2019).

Se ha dividido este trabajo en cinco secciones, además de la introducción. En la primera parte se desarrolla un análisis conceptual basado en las movilidades rurales. En la segunda sección se describe el enfoque metodológico utilizado para el estudio de movilidades mapuche-williche. En la tercera sección se presenta una síntesis del trabajo de campo desarrollado en la comuna de Puyehue. En el cuarto apartado se exhiben categorías emergentes de la fase en terreno. Finalmente se presenta una reflexión en torno a la movilidad cotidiana y su relación con aspectos socioculturales propios del sur de Chile.

\section{Movilidades rurales e indígenas en constante interacción}

Las movilidades rurales ofrecen nuevas perspectivas acerca de la compleja interacción entre las movilidades y las dinámicas de los espacios rurales, aportando nuevas luces en las discusiones respecto de movilidad cotidiana. En la Gales rural, Milbourne y Kitchen (2014) exploraron las complejidades espaciales y temporales de la migración rural, destacando: a) las diferentes escalas, direcciones y temporalidades que caracterizan el movimiento de la población en las áreas rurales, b) formas en que las personas que viven en lugares rurales practican las movilidades, centrándose en sus experiencias de viajes y sus dificultades, y, c) la creciente importancia de las formas virtuales de movilidades dentro de las vidas de los residentes rurales. Estos elementos parecen fundamentales cuando se quiere definir y problematizar las movilidades no metropolitanas.

Son las diferentes movilidades (etarias, por género o etnia) las que producen las complejidades del mundo rural. En el caso de los pueblos indígenas, son escasas las investigaciones que examinan las implicancias de la movilidad cotidiana para las políticas territoriales, la indigenidad y el cambio social en las áreas rurales (Sletto, 2016). A causa de este vacío, resulta necesario analizar la movilidad rural desde una perspectiva indígena que desestabilice las definiciones estáticas de la ruralidad (Panelli et al., 2009) y otorgue nuevos marcos de interpretación de las categorías espaciales de los pueblos originarios.

Debido a las deficientes condiciones de accesibilidad a servicios básicos, educación y falta de oportunidades laborales, gran parte de la población indígena migra a la ciudad para escapar de la pobreza, pero también para aprovechar oportunidades ambientales, políticas y económicas (Sletto, 2016; González-Parra y Simon, 2008) sin necesariamente desvincularse de sus territorios de origen (CEPAL, 2014). Sin embargo, existen otros patrones migratorios que responden a una movilidad mediante las fronteras internacionales o jurisdiccionales en territorios históricamente habitados por pueblos indígenas (Ramos, 2018; Contreras, Tapia y Liberona, 2017). Mientras que, en otros casos, las metrópolis atraen población indígena sin responder necesariamente a la existencia de 
territorios tradicionales separados por dos o más Estados (CEPAL, 2014).

El concepto de territorio se ha vuelto fundamental, en especial para las demandas de los pueblos indígenas latinoamericanos relacionadas con las políticas del espacio, la asignación de derechos y la concepción de la autoridad política (Bryan, 2012). En este contexto, han surgido demandas por comprensiones del espacio situadas culturalmente con base en enfoques relacionales que desafían categorías del territorio como espacio cartográfico (Bryan, 2012; Echeverri, 2005). Esto se torna de suma importancia en las agendas políticas, considerando el legado del despojo de tierras indígenas, la creación de reservas y reducciones indígenas y procesos de colonización que continúan teniendo serias repercusiones en las movilidades indígenas (Huiliñir-Curío, 2018; Sletto, 2016; Norman et al., 2015).

La literatura crítica pertinente a movilidades indígenas considera las múltiples influencias de procesos globales y modernos pasados y presentes (Carey y Lydon, 2014). En efecto, los pueblos indígenas han respondido a estas situaciones desarrollando nuevas formas de supervivencia y de movilidad (Carey y Lydon, 2014), trazando redes de relaciones e identidades sociales que recurren a la memoria para conceptualizar nuevas y complejas formaciones territoriales, que abarcan tanto su asentamiento actual como sus sitios de origen (Gordillo, 2004, 2011; Sletto, 2016).

La memoria es un importante recurso narrativo que permite comprender las relaciones complejas entre la producción de las identidades indígenas y nuevas formaciones socioespaciales. De acuerdo con Mendoza (2005), la memoria se construye con base en narraciones que forman discursos "construidos sobre un conjunto de saberes compartidos" (Lindón, 1999: 300) y modos de organizar experiencias pasadas y presentes que llenan de sentido al mundo. Legg (2005) plantea que los procesos de memoria y olvido no son solo específicos a los acontecimientos históricos y su interpretación, sino también al entorno y la inclusión o exclusión de recuerdos en él. De esta manera, la memoria no debe estar ausente como categoría analítica de las movilidades y las identidades cambiantes de los pueblos indígenas, poniendo de relieve el activo ejercicio de restauración de memorias en los procesos de configuración de nuevas espacialidades (Ramos, 2014).

Si nos referimos al pueblo mapuche, se observa que este siempre ha sido móvil. Desde antes de la conformación de los Estados nacionales de Chile y Argentina (finales siglo XIX), la mayoría de las personas mapuches migraban estacionalmente entre las costas hacia los territorios interiores en busca de alimento y refugio, cruzando la cordillera hacia las pampas de Argentina (Bello, 2011).

Gundermann, González y De Ruyt (2009a; 2009b) examinan la migración y movilidad campesina y mapuche desde las regiones de $\mathrm{La}$ Araucanía, Los Ríos y Los Lagos a la Patagonia argentina durante la segunda mitad del siglo XX, resaltando las persistencias y cambios de estas dinámicas en función a factores económicos y los vínculos entre Chile y Argentina. Desde un enfoque de las prácticas de movilidad mapuche, Godoy (2017) caracteriza la movilidad mapuche por los caminos cordilleranos por medio de relatos orales, destacando las prácticas asociadas a dichos desplazamientos, así como la utilización de troncos ahuecados usados como espacios de refugio, el uso de canoas o wampos, un sistema de señales de humo o fütrunkütral. Del mismo modo, Huiliñir-Curío (2015 y 2018) ha explorado las diversas formas de movilidades mapuches en la cordillera de los Andes, a partir del análisis de las categorías espaciales de las huellas indígenas asociadas a la movilidad mapuche-pehuenche, y posteriormente, los paisajes producidos por las movilidades de una comunidad mapuche situada en Curarrehue (Huiliñir-Curío, 2018).

Respecto de las geografías del pueblo mapuche, el concepto de tuwün se torna clave en nuestra investigación, que puede traducirse como "proceder" o "lugar de origen" (Di Giminiani, 2012: 100). El tuwün es la ubicación geográfica compartida, desde donde se origina una persona y su patrilinaje (Di Giminiani, 2012). Vidal et al. (2017): "tuwün es el espacio territorial de donde procede y gracias al cual existe un ce (persona) particular. Puede ser el lugar donde la persona habita, aunque un ce puede haber mudado su residencia y estar viviendo fuera de su tuwün" (Vidal et al., 2017: 105).

El tuwün es relevante porque da cuenta de nociones actuales de propiedad de la tierra y sus trayectorias. A su vez, la noción de tuwün es 
empleada por las personas mapuches para evitar la migración y fortalecer el sentido de pertenencia a un lugar, condición necesaria en la que se funda la identidad mapuche: el tuwün influye en el comportamiento -y movilidad- de la persona, articulando diferencia y mismidad (Di Giminiani, 2012).

La valoración del pasado es otro elemento por considerar de las prácticas discursivas mapuches contemporáneas, a partir de ello es posible comprender la forma del ser mapuche (Vidal et al., 2017). Sin ánimo de pretender definir qué es o no es ser mapuche, parece relevante considerar esta mirada retrospectiva que define muchas de las acciones y trayectorias personales ligadas al territorio de origen, presentes en las prácticas discursivas mapuches. Como señala López et al. (2014), aunque mucha población indígena no resida en sus lugares de origen (la mayoría rurales), el territorio y la restitución de sus tierras despojadas continúa siendo un importante elemento de continuidad histórica.

\section{¿Cómo aproximarse a movilidades no metropolitanas?}

Este estudio se distancia de la comprensión tradicional de la movilidad cotidiana debido a que los participantes residen en espacios rurales caracterizados por el desarrollo de desplazamientos graduales y planificados en un tiempo-espacio no necesariamente diario. Se trata de movilidades estacionarias pero intensas que obligan a desplegar técnicas que consideren las particularidades de esta movilidad rural y su relación con aspectos rítmicos y territoriales.

El trabajo de campo se desarrolló durante el otoño del 2018 en localidades como El Caulle, Porvenir, Santa Elvira, Nadi, Pichi Dama, Entre Lagos y Las Parras, estableciendo contacto con 20 personas que se transformaron en los participantes de la investigación. Todos/as ellos/as residen en comunas y localidades de Los Lagos, específicamente en Puyehue o sus cercanías, sus edades fluctúan entre 19 y 77 años, y las principales actividades que realizan se relacionan con trabajo agrícola, actividades domésticas, estudios de nivel medio/superior y dirigencia social. Los criterios de selección están relacionados con los objetivos que persigue una investigación de mayor extensión que tiene por finalidad analizar las prácticas y experiencias de movilidad en ciudades del sur de Chile con énfasis en aspectos experienciales del viaje y la configuración de la movilidad en su ambiente. Resulta necesario aclarar que el material presentado aquí solo incluye personas pertenecientes al pueblo mapuche-williche, lo que constituye un factor clave en las categorías analíticas que serán presentadas en las próximas páginas. Esta elección se fundamenta en la necesidad de relevar aspectos de la movilidad cotidiana que han sido escasamente tratados en nuestro país, como son las prácticas de movilidad cotidiana de la población mapuche-williche.

Por otra parte, es necesario mencionar que previo a la inmersión en el trabajo de campo, se efectuó una revisión de las principales técnicas de investigación cualitativas que se han desarrollado en los últimos años en Chile para analizar las prácticas de movilidad cotidiana. Estas herramientas se han desplegado básicamente en contextos urbanos y permiten analizar la movilidad matizando técnicas con características etnográficas como el sombreo (Jirón, 2011; Imilan et al., 2015); entrevistas de viaje (Avellaneda y Lazo, 2011; Lazo y Carvajal, 2018; Huiliñir-Curío, 2015; Salazar, et al., 2017; Lazo y Calderón, 2014); caminatas (Salazar et al., 2015; Huiliñir-Curío, 2018; Jirón et al., 2016) y mapas mentales y dibujos (Iturra y Jirón, 2016; Riquelme y Riquelme, 2018). Esta revisión conllevó a seleccionar, producto de las características de los participantes, técnicas que permitieran profundizar aspectos más específicos de su movilidad. Para ahondar en ello se desplegaron tres herramientas cualitativas: entrevistas semiestructuradas, observación participante y mapas mentales.

Las entrevistas fueron confeccionadas a base de un guion temático que trató dimensiones de la movilidad rural como objetos de viaje, accesibilidad, medios de transporte, experiencias de viaje y motivos de viaje. En segundo lugar, la observación participante, percibida como un proceso donde se participa observando y se observa para participar, fue utilizada para realizar descubrimientos de manera crítica en relación con los viajes y desplazamientos de las personas. Esta técnica implicó conocer rutas, medios de transporte, espacios íntimos y lugares representativos de la movilidad mapuche-williche. Los dos puntos anteriores fueron acompañados de la realización de mapas mentales, que permitieron conocer cómo 


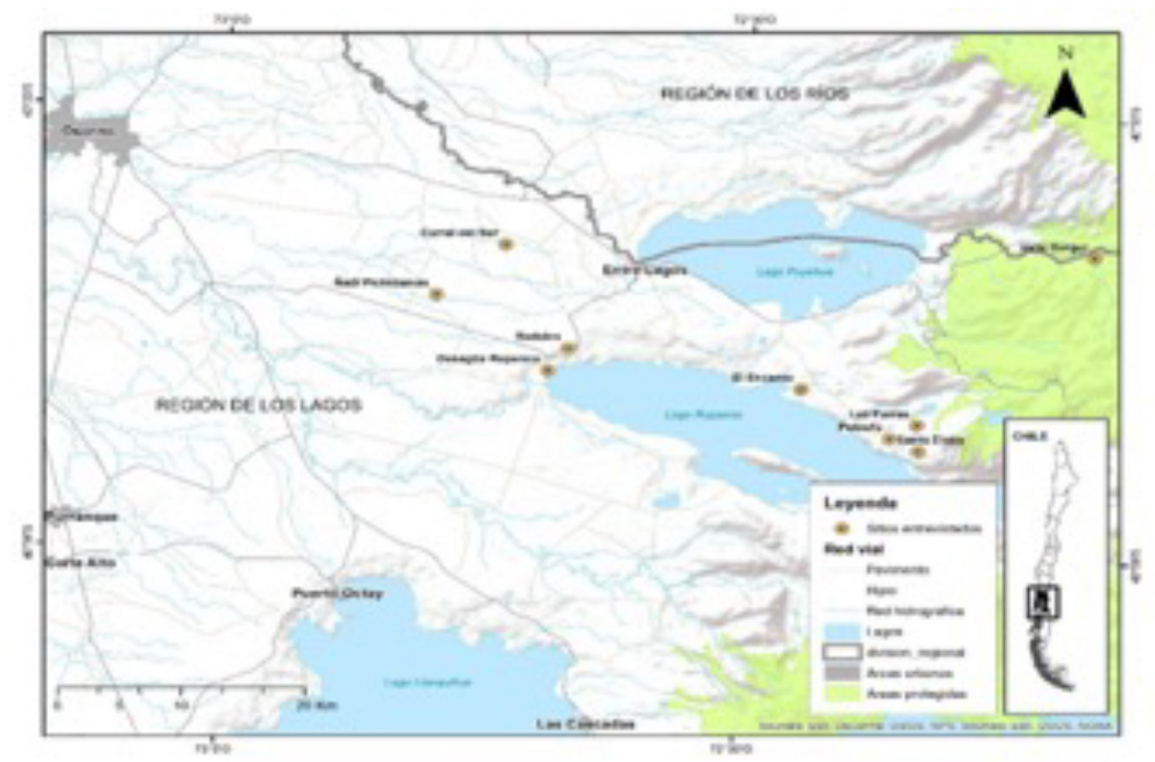

Figura 1. Mapa de la zona de estudio.

Fuente: Archivo de autores.

los participantes representan el espacio habitado y construyen el territorio rural. Finalmente, se realizaron relatos etnográficos con algunos de las personas, al objeto de seguirlos en sus rutinas diarias de movilidad, conocer sus rutas, ritmos, tiempos y actividades, al mismo tiempo observar el significado que le daban a sus espacios de movilidad.

\section{La movilidad como forma de habitar la ruralidad}

A continuación se presenta un relato de carácter etnográfico que permite conocer las actividades y lugares que se circunscriben al ambiente cotidiano de una habitante mapuche-williche de la comuna de Puyehue. Se presentan sus lugares, rutas y quehaceres cotidianos.

\section{Las rutas de María: explorando movilidades mapuche-williche en la ruralidad}

María, 48 años, mujer mapuche-williche, habita en el valle Gol Gol, ubicado en la comuna de Puyehue. Este sector es un valle cordillerano situado entre el volcán Puyehue y el cerro Los Derrumbes, donde el acceso es por la ruta internacional 215-CH. No existe ningún tipo de servicio de transporte público en el sector, por lo que los habitantes tienen que viajar a Entre Lagos para acceder a los distintos servicios, personas y lugares.

María se desplaza a trabajar, como educadora intercultural en la escuela de las Termas de Puyehue, en un vehículo que acaba de adquirir. Además de este trabajo, María es miembro activo de las reuniones del Consejo de Comunidades Mapuche-Williche de la comuna.

Viajamos desde Osorno hacia Entre Lagos en vehículo con el objetivo de dirigirnos hacia la casa de María. La ruta está en mal estado, con bastantes baches y de alta complejidad de maniobras producto de las distintas curvas cerradas que posee.

Al llegar, comenzamos a conversar de su vida mientras desayunábamos. María y Marcos (su esposo), dispuestos de su machete y sus perros Black y Jack, nos invitan a acompañarles en las labores que realizan en el campo, donde nos facilitan un par de botas para así cruzar los distintos esteros que hay en el camino.

Tras caminar 100 metros, nos encontramos con un cerco con tranca de metal, María nos comenta que esta tranca se encuentra cerrada desde el año 2010, cuando comenzaron las disputas entre el Club Andino y la familia de María por el terreno en donde se encuentra su casa y parte de 


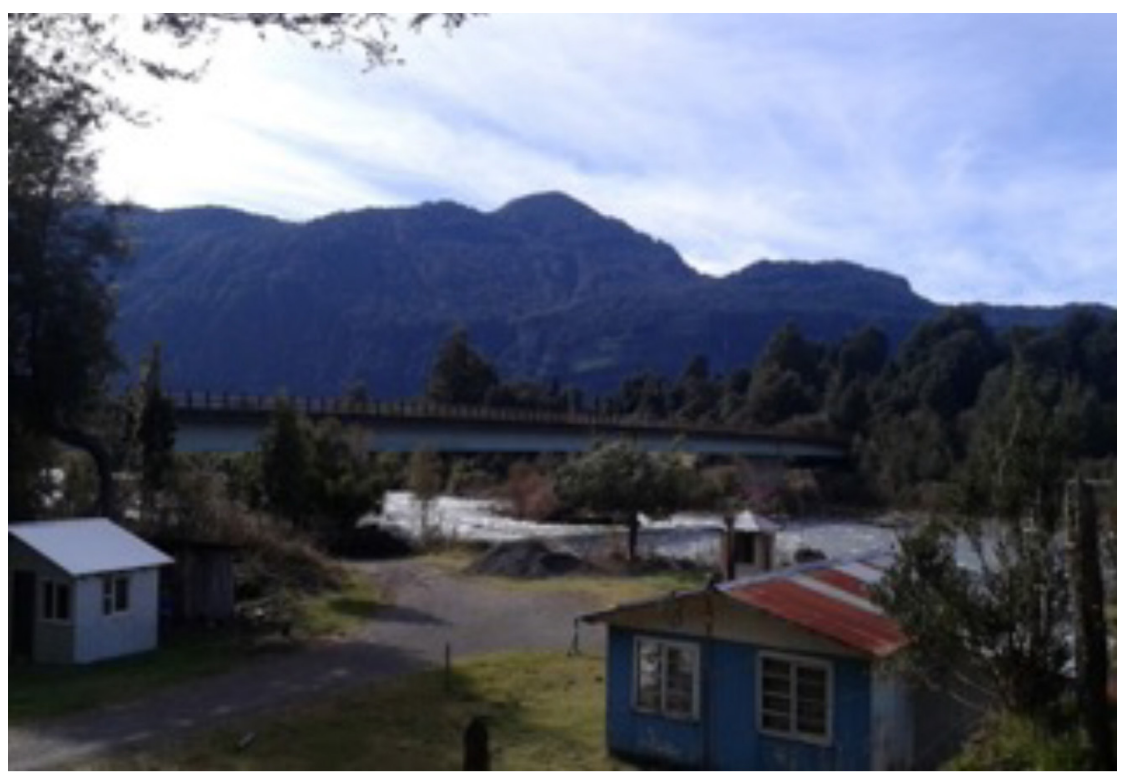

Figura 2. Sector Gol Gol. Descripción: sector de residencia de María.

Fuente: Archivo de autores.

su campo que colinda con esta institución. Por lo que para ir a revisar sus animales María y su familia tienen que saltar este cerco con tranca.

Al poco tiempo de avanzar, María nos comenta que esta ruta que realiza para llegar a las pampas donde se encuentran sus animales pastando (vacas, terneros, novillos y toros) es la ruta 215 antigua, que fue utilizada a su vez como ruta ancestral por su familia (Queulo) para movilizarse hacia Entre Lagos y Villa La Angostura (Argentina). Esta ruta se encuentra en buen estado, debido a que se utiliza exclusivamente para el tránsito de personas a pie. Llegamos a otro cerco con tranca, rústica y de alambre de púas, típica de los campos del sur de Chile, la que demarca el inicio del campo de María, y al mismo tiempo se dejan ver los primeros animales. Marcos menciona que los animales que se encuentran en el campo vagan libremente por todo el este y el bosque aledaño.

Luego de caminar alrededor de cuatro kilómetros, nos encontramos con un letrero que marca el inicio del Parque Nacional Puyehue, a un kilómetro están los claros donde se encuentra el resto de los animales y un corral que utilizan para interactuar con estos. María menciona que estos claros lo realizaron sus tíos, y estos vivieron en aquel lugar hasta el terremoto de 1960 y la erupción del volcán Puyehue, dejando abandonado el lugar debido a los derrumbes que se produjeron en el cerro. Para María es un lugar de gran importancia debido a que es el sitio donde sus ancestros vivieron, por lo que se percibe en el lugar una gran carga emocional. María también menciona que es muy difícil tener animales en el sector, ya que no pueden acceder con un tractor o una carreta para forrajear, solo pueden llevar animales pequeños a una pampa cercana a su casa, ya que es más sencillo levantarlos por un cerco con tranca cerrada.

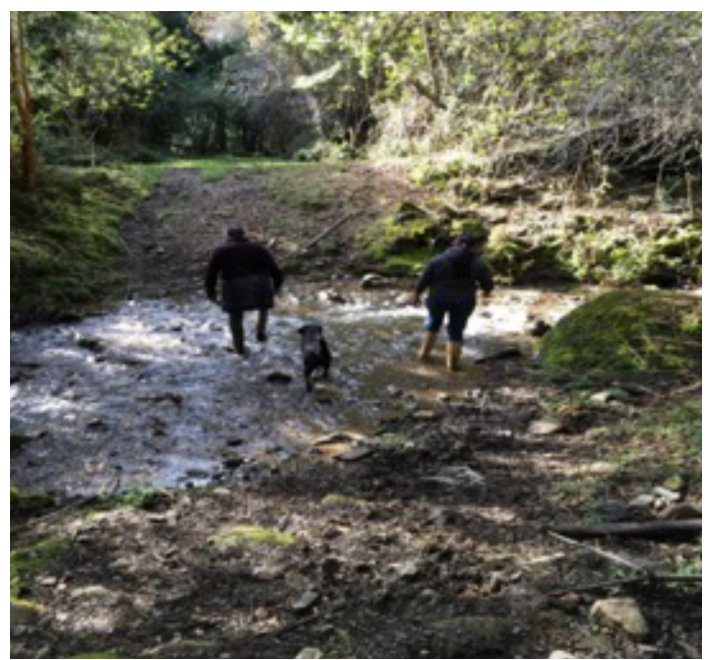

Figura 3. María, Jack y Marcos caminando por sus terrenos.

Fuente: Achivo de autores. 


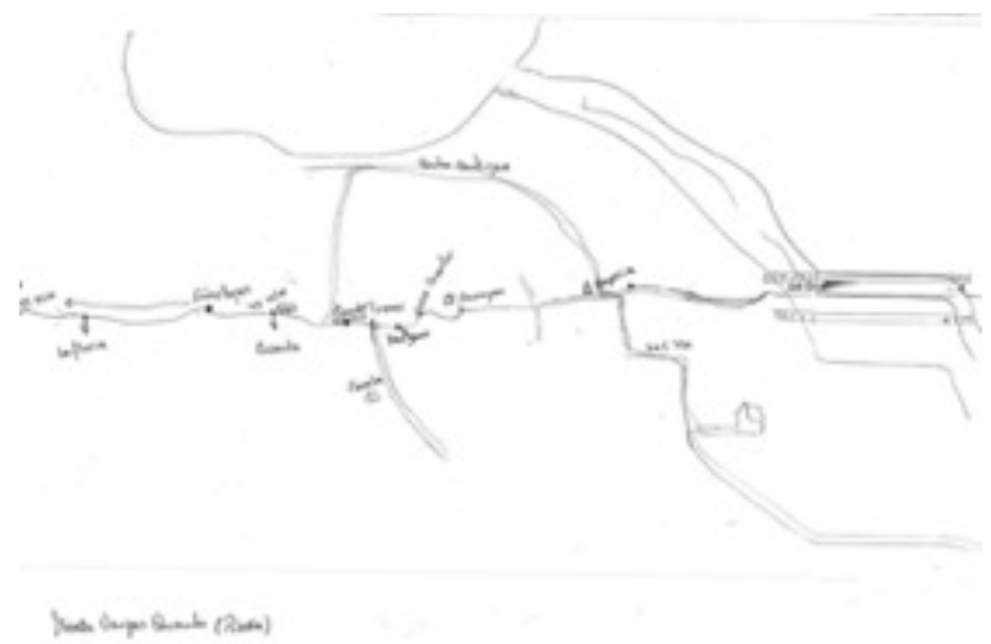

Figura 4. Mapa mental elaborado por María. Se distinguen diversas rutas y lugares que conforman su espacio de vida cotidiano.

Fuente: Archivo de autores.

Comienza a caer la tarde y decidimos emprender el viaje de vuelta, llegamos alrededor de las 13: 15 horas. María sirvió el almuerzo y nos convidó muday que había preparado especialmente para refrescarnos.

Luego de almorzar junto con María y Marcos, caminamos por un camping a la orilla del río, lugar donde recolectan leña para su consumo. Este camping lo tienen habilitado con ducha y baño, todo construido por ellos. Más adelante, María nos muestra un quiosco en que el verano vende a los turistas tortillas, miel y otros productos que ella realiza. María menciona que estos turistas aparecen por las referencias de la alta calidad de sus productos.

Ya siendo las cuatro de la tarde, regresamos a Osorno. Nos despedimos de María, que se dirige a la casa de su madre con el objetivo de acompañarla durante lo que queda del día.

\section{Construyendo categorías de análisis desde lo mapuche-williche}

Para analizar la movilidad de los habitantes mapuche-williche de las localidades de la comuna de Puyehue se establecieron nueve categorías que emergieron del trabajo de terreno con la población mapuche-williche del sector. Las categorías son: 1) Divisiones y permisos; 2) El viaje y el caminar; 3) El comercio y las fiestas; 4) Los objetos; 5) Tüwün; 6) Malla territorial; 7) La reciprocidad y las estrategias; 8) El antes (la memoria) y 9) Tensiones.

La primera categoría tiene relación con lo que los habitantes llaman divisiones y permisos. Esta categoría remite a una concepción del territorio particular que lo concibe como un lugar único y significativo de movilidad que se requiere para estar en armonía con el entorno, por lo que las divisiones y los permisos van a ser de importancia en la lógica del movimiento. El williche pide permiso para cruzar de un territorio a otro, que está muchas veces delimitado por las aguas, ríos y saltos. Ello marcará para estos habitantes una diferencia significativa con lo que es el movimiento del winka, quien no considera esa conexión con el territorio que habita. $\mathrm{Al}$ respecto, uno de los entrevistados sostiene:

"Leufü es un río o un salto donde hay un ngen, eso es una parte de espiritualidad mapuche, a eso se le llama Leufü (...) si usted no pide permiso, a usted lamentablemente el agua lo va a dejar en un par de segundos estilando de mojado, lo corre, lo echa y un viento que sale como que lo sopla así, heladísimo" (Juan).

El permiso o ekuwün también expresa una relación respetuosa con elementos de la naturaleza, preservados por un ngen o espíritu cuidador de cada territorio. Como señalan Quintriqueo et 
al. (2014), el respeto a cada ngen o fuerzas sobrenaturales aseguran el bienestar colectivo e individual, evitando enfermedades o accidentes en lugares considerados riesgosos.

"Se iba al kütral o se hacía en el agua, esos eran los permisos para poder salir porque se supone que donde tú ibas traspasabas agua y traspasabas tierra, o sea, esa es la lógica del mapuche. Usted sabe que la división del mapuche es el agua, eso marca territorio, no nos marca la tierra, la tierra es el sostén, el arraigo mapuche, pero el agua no, el agua es división de un territorio a otro y traspasar el agua para nosotros es súper importante" (Melita).

La segunda categoría hace alusión al viaje y el caminar. Dentro de las comunidades rurales y también indígenas, el viaje ha estado siempre presente en la memoria, viéndose como algo positivo y parte de su identidad. Viajes que no siempre se dan en un radio acotado (territorio de proximidad), muchas veces traspasan los límites administrativos de las regiones (van de cordillera a mar) y también se efectúan entre fronteras (viajes a Argentina). El viaje y el caminar es percibido por muchos como parte del legado de sus ancestros: se camina para alimentar a los animales, visitar a los vecinos, ir al pueblo y recoger los alimentos. Esta misma idea se relaciona muy bien con el concepto de "líneas y malla" planteada por Ingold $(2011,2012)$, que se retoma en esta investigación.

\begin{abstract}
"Viajaban mucho los lamienes y todavía viajan mucho cuando hay que organizar algo, pero obviamente como ahora está un poquito más cómodo o con mejor movilización o los caminos se han arreglado, pero igual se viaja harto" (Catalina).
\end{abstract}

La tercera categoría permitió entender los motivos de los desplazamientos relacionados con prácticas tradicionales del mundo mapuche, algunas más frecuentes que otras. En efecto, el comercio y las fiestas fueron y son motores de movilidad para estos habitantes. Asoman categorías como trafkintu que es el comercio de semillas y alimentos, que mueve a muchas familias a distintos territorios de la región para comerciar, desplazándose varios kilómetros de distancia. Dentro de esta práctica, las mujeres mapuches juegan un rol importante como curadoras de semilla que preservan e intercambian con mujeres de otros territorios.

El trafkintu también es un ejercicio de memoria que revitaliza alianzas y lazos con otros territorios. El Wetripantu, ceremonia de año nuevo mapuche, también es motivo de desplazamiento hacia la comunidad de origen con el objeto de celebrar y compartir. Esta celebración se realiza durante el solsticio de invierno en el hemisferio sur y marca una etapa de renovación de la naturaleza y también espiritual. La minga, actividad social que consiste en un trabajo realizado en comunidad, en la que se comparte y se celebra, mientras se traslada una casa de un lugar a otro, se cosecha papa o se construye una ruka o casa tradicional mapuche. Por último, el palin, que es un juego tradicional mapuche, comunitario y de importancia sociopolítica, en el que se reúnen dos o más territorios mapuches (Lof). Se observa entonces cómo las festividades y el comercio van asociados a prácticas de movilidad cotidiana específicas.

"Sí, para El Caulle, para El Encanto, para el Llano, ese es nuestro afán ya sea del Trafkintu de lo que fuese, de conocimiento, de alguna ceremonia espiritual y también el Trafkintu de alimentos, semillas y esas cosas" (Celia).

"Porque yo soy de la tierra, amo la naturaleza y soy parte de ella, ella me tranquiliza a mí, eso me pasa con la naturaleza y por eso voy a las ceremonias, al We Tripantu, me desplazo buscando aquello que nosotros perdimos en algún momento lo trato de buscar con mis lamienes" (Celia).

"Somos bien viajeros, sobre todo cuando hay ceremonia ahí somos bien buenos para viajar, para hacer mingas, para ayudar a los lamienes igual" (Catalina).

La cuarta categoría hace alusión a los objetos, y se relaciona con algunas materialidades que son llevadas en el viaje por las comunidades. Acá aparecen el termo y el mate como elementos culturales y diferenciadores, como también la 
vestimenta tradicional, que es un elemento que le da una connotación particular y sentido al viaje. Junto con estos elementos, que podríamos denominar tradicionales, convive otra materialidad a partir del uso del celular, como elemento esencial en la organización: previaje, durante el viaje y postviaje. Muchas de estas personas conciben de manera dificultosa su vida sin este dispositivo tecnológico, sobre todo considerando el contexto geográfico en el que habitan.

"Cuando uno puede generalmente venirse en el vehículo y ahí entonces uno se coloca un poncho, su atuendo que de repente en caso de, lo puedo utilizar y lo ando trayendo y el termo y el mate... claro el termo y el mate, eso siempre tiene que andar en la mochila" (Cristina). "Cuando voy como mapuche siempre llevo un mate, siempre estoy llevando yerbita ¿me entiende? Para compartir, en cambio, como winka, no, porque yo voy a hacer un papeleo, voy a buscar mi mercadería por decirlo de alguna manera y de ahí me vengo" (Melita).

"Primeramente, el celular porque es una herramienta de trabajo como para poderme comunicar, eso es cuando yo pueda llamar y tenga plata (risas)" (Cristina).

La quinta categoría se refiere al tüwün, o lugar de pertenencia, y tiene relación con el apego de las comunidades respecto de su territorio como un lugar con el que se entretejen vínculos afectivos e identitarios. Esto se relaciona con la importancia que tiene el lugar de pertenencia y el medio ambiente como actantes que permiten una movilidad que a veces sobrepasa el territorio próximo. Para algunos, la movilidad se entiende como un medio para visitarse, para comunicarse con los otros. El tüwün será visto como un territorio de proximidad, ese anclaje que permite la movilidad.

"Acá se vive totalmente distinto, son otros ritmos, el medio que te presiona, que te contamina el ambiente, el ruido que tienes que ver, la cantidad de gente que tienes que aguantar, entonces todo eso es estresante, el ritmo de vida es estresante, entonces ahí se generan las complicaciones, bueno y como mi ascendencia es mapuche obviamente igual no hay donde perderse con el campo, uno es parte de la tierra" (Ricardo).

El tuwün también se vincula con lugares significativos para las personas williches, relacionadas con una historia familiar o del territorio. Esto da cuenta que el tüwün responde a una historicidad presente en la memoria de los entrevistados/as, ampliando las escalas de lo que se comprende como el territorio próximo. Un ejemplo de ello es el siguiente relato:

"Yo encuentro que El Caulle es un lugar
importante; yo creo que espiritualmente
está preparado; yo creo para las rogativas
a parte está al lado de la cordillera, siento
que es importante y bueno Kintuante que
está en el lado de Mantilhue para el lado
de Carimallin ahí está la machi también,
entonces creo que esos lugares son im-
portantes" (Jocelyne).

La sexta categoría se refiere a la malla territorial. Se evidenció que, para estas comunidades, los senderos, caminos y huellas de antaño también van construyendo sus identidades en la memoria en torno a la movilidad.

En esta categoría emerge el concepto de rüpu que se relaciona con las escalas y paradas que cada tanto los habitantes hacen antes de llegar a destino: visitar al vecino, ir a intercambiar alimentos, saludar a un amigo, tomar el bus al pueblo $\mathrm{y}$ volver. Ese viaje construido como una serie de hitos y escalas, implica aprender el camino en la práctica desde temprana edad, a partir de esto se reactualiza este conocimiento corporizado del territorio en torno a huellas y líneas dejadas por los habitantes.

"Sí, y tenían sus propios senderos que hasta los días de hoy todavía existen porque, por ejemplo, hay un sendero por Santa Elvira, un camino muy antiguo, tiene un nombre católico, pero para nosotros es un camino ancestral que comunicaba del Puel Mapu hacia este lado, las lamienes del Puel Mapu iban hacia allá y nosotros íbamos con ellos, mapuche de allá, del otro sector" (Melita). 
Recorrer cada rüpü y lugares de importancia familiar, constituye una práctica de fortalecimiento de los lazos familiares. Mediante los viajes por las huellas se transita por las trayectorias de los movimientos migratorios y circulatorios de cada familia en la cordillera y, a su vez, se establecen nuevas interacciones en cada sitio destinado al descanso.

Asimismo, lagos y pasos cordilleranos también forman parte de ese imaginario que se reactualiza. Acá, la referencia a lo no humano, como lagos, cordilleras y otros elementos naturales, son muy importantes en la concepción del movimiento. Lagos y pasos cumplían variadas funciones, las que se supeditaban a las condiciones climáticas y geográficas, como puentes naturales vitales para los viajes por la cordillera.

Esta categoría se relaciona con la idea de rüpü, debido a que forman parte de la malla de líneas que conforman la territorialidad williche, develando una compleja red de intercambios y prácticas particulares en el territorio, integrados al paisaje recorrido en cada huella:

"Generalmente arriba en Las Termas de Puyehue, por ahí hay un paso, hay como dos o tres pasos, inclusive la gente ahí esperaba cuando estaba malo el tiempo tenían como un hoyo, una cueva y ahí esperaban, hacían fuego... qué sé yo; y a algunos a veces les tocaba alojar ahí para pasar porque el lago estaba bravo a veces" (Celia).

Respecto de la séptima categoría, reciprocidad y estrategias, es importante señalar que da cuenta de la existencia de movilidades colectivas y organizadas en función de ayudar al otro. Estas comunidades se apoyan y buscan formas de mejorar sus movilidades en un contexto de marginación y pobreza. Este elemento es común en las comunidades rurales y también viene del pasado, dándole un sentido a las movilidades actuales.

"Yo he visto tres métodos porque me ha tocado vivirlos, por ejemplo, la locomoción que tienen a veces a diario, no todos los sectores. La otra es que le van a tocar al vecino que tiene locomoción y algunos hacen intercambio, hacen trafkintu, por ejemplo, de papas o yo te traigo bencina para la motosierra, o yo te traigo esto" (Melita).

"Por lo que yo he visto, más que nada viajan en un vehículo, arriendan vehículo o en el bus, pero siempre he visto más gente a dedo. Hay gente que entra a trabajar a dedo, hay gente que tienen que ir a Osorno y se van a dedo un trayecto, por ejemplo, donde no hay locomoción a dedo de ahí ya cuando llegan acá a Entre Lagos y ahí se van en bus" (Jocelyne).

Antes del viaje, como octava categoría, se ha identificado de manera transversal, ya que hace referencia a la memoria, al "cómo se viajaba antes". Esta dimensión permite darle un sentido al presente de la movilidad que supone sobrellevar las dificultades. Si bien las movilidades actuales parecen ser más rápidas en tiempo y más cómodas en transporte (se camina menos y casi no se utilizan animales) siguen existiendo importantes problemas de aislamiento y conectividad que parecen verse enfrentados de mejor manera al compararse con el pasado y con la concepción particular que tienen del viaje estas comunidades.

"Antiguamente me contaba mi mamá que su abuelo de Mantilhue, tenía que venir en bote y por lo que escuche aquí en Entre Lagos había una barcaza igual, por eso es que hay como muelles" (Catalina). "Se hacía todo ese recorrido, de a pie, a caballo, en bote, eran boteros antes" (Celia).

La última categoría tiene relación con la tensión, con esa dualidad siempre presente entre ser williche y chileno. En relación con el viaje, sus significados y experiencias, se evidenció una diferencia entre lo que significa viajar, aludiendo a los objetos, las formas, la vestimenta, y el significado mismo del desplazamiento williche en comparación con un viaje simple como chileno o winka, donde las formas y los significados tienen otro sentido. Eso genera una tensión a nivel del discurso y de la práctica misma del desplazarse, relacionadas con la construcción de la alteridad.

"Yo ayer vi eso porque pasó mucha gente en la ruta, entonces uno ve el sentir de las 
personas que le molesta la presencia mapuche, pasa eso, yo he sentido todas esas cosas, las he visto también, las he vivido y la diferencia de andar con ropa natural como todos y andar con su atuendo" (Norma).

"Se ha olvidado de lo que es vivir como mapuche porque no es lo mismo viajar a patita, sentir la tierra que viajar en locomoción en donde yo insisto no te saluda nadie, nadie hace nütram con otro, todos andan con su celular y esas cositas (Jaime).

\section{Conclusiones}

A partir del conocimiento de las prácticas de movilidad cotidiana de los habitantes mapuche-williche, se ha podido evidenciar cómo el componente sociocultural hace que las prácticas de movilidad cotidiana no solo tengan que ver con cuestiones como distancias, tiempos y modos de transporte, sino que también -y por sobre todo- con una concepción y cosmología del territorio particular, donde categorías como tüwün, rüpu, permisos, trafkintu, memoria del viaje, etc., cobran fuerza y hacen que la experiencia de moverse sea diferente a los habitantes urbanos o rurales no mapuches, dando cuenta de aquello denominado la movilidad en su ambiente. Se trata de comprender las movilidades rurales como una circularidad relacional donde convergen prácticas, experiencias, significaciones y materialidades en un contexto sociocultural propio de este territorio.

Al conceptualizar acerca de la dimensión indígena de la movilidad, es posible visualizar dinámicas inherentes a las ciudades del sur de Chile donde la matriz territorial-cultural mapuche juega un rol importante en las prácticas, experiencias y significados otorgados a la movilidad. De esta manera, las conexiones con el ámbito urbano siempre han estado presentes en estas poblaciones, generándose una territorialidad en red (Huiliñir-Curío, 2015) o territorios circulares, producto de las conmutaciones cotidianas.

Se cree en la necesidad de problematizar el territorio desde una perspectiva relacional que involucre las prácticas, experiencias y objetos desde la representación de las dinámicas territoriales, las prácticas socioculturales y el diseño de ciudades y carreteras. No obstante, la mayoría de estas movilidades tienen la característica de darse en la urbs. Es menester visibilizar las movilidades menos urbanas de la civitas, que en el caso de Puyehue comprende no solo al ámbito físico-natural y al espacio de vida, sino que también el "espacio vivido" y "practicado" (Huiliñir-Curío, 2015), cuestiones fundamentales para el mundo mapuche y su movilidad.

La movilidad cotidiana constituye un elemento identitario para el mundo williche, en donde se integran nuevos artefactos y otros medios para desplazarse actualmente, pero que no afectan esa idea de movilidad diferente al de los no williches. Resulta interesante develar por qué el celular o la camioneta, por ejemplo, son indispensables hoy, pero estos elementos no imposibilitan la sensación de sentirse williche. Asimismo, las dificultades que implica viajar se ven más acentuadas con las condiciones materiales y las desigualdades territoriales. Esto obliga a migrar a la ciudad o hacer viajes laborales complejos, por la necesidad de no dejar el tüwün, muy ligado a un discurso identitario.

La emergencia de las nueve categorías y el relato etnográfico aquí presentado, resultan de suma relevancia para analizar las movilidades rurales y su componente indígena en el sur de Chile, considerando ritmos, espacios y actividades propias de estos territorios, lo que conlleva a un distanciamiento respecto de la perspectiva teórica y metodológica del estudio de la movilidad netamente urbana. Esto exige la creación de nuevas estrategias para analizar las experiencias cotidianas de los habitantes de la ruralidad con tradiciones, costumbres y prácticas cotidianas particulares.

\section{Agradecimientos}

A CONICYT- FONDECYT por el financiamiento del proyecto 111710128 . De la misma forma, los autores reconocen a Giacomo Bavestrello y Antonio Vargas por la colaboración prestada en la recolección de información durante el trabajo de campo. Asimismo, agradecen a los revisores anónimos por sus valiosos comentarios. 


\section{Referencias Citadas}

Avellaneda, P. y Lazo, A.

2011 Aproximación a la movilidad cotidiana en la periferia pobre de dos ciudades latinoamericanas. Los casos de Lima y Santiago de Chile. Revista Transporte y Territorio, 4.

Bello, A.

2011 Nampülkafe, el Viaje de los Mapuche de La Araucanía a las Pampas Argentinas. Ediciones UCT, Temuco.

Bryan, J.

2012 Rethinking territory: social justice and neoliberalism in Latin America's territorial turn. Geography Compass, 6 (4): 215-226.

Carey, J. y lyndon, J.

2014 Introduction Indigenous Networks: Historical Trajectories and Contemporary Connections. En Indigenous Networks. Routledge, London.

Castelleti, J.; Villarroel, M.; Almendares, H., y Mercado, P.

2019 Historia del paisaje rural entre las familias de Paposo y Taltal. Diálogo Andino (58): 77-88.

CEPAL (Comisión Económica para América Latina y el Caribe)

2014 Los pueblos indígenas en América Latina. Avances en el último decenio y retos pendientes para la garantía de sus derechos. Cepal, Santiago.

Contreras, Y.; Tapia, M. y Liberona, N.

2017 Movilidades y prácticas socioespaciales fronterizas entre Arica y Tacna. Del sentido de frontera a la transfrontericidad entre ciudades. Diálogo Andino (54): 127-141.

Di Giminiani, P.

2012 Tierras ancestrales, disputas contemporáneas: Pertenencia y demandas territoriales en la sociedad Mapuche rural. Ediciones UCT, Temuco.

Echeverri, J.

2005 Territory as body and territory as nature: Intercultural dialogue. Eds. A. Surrallés, PG Hierro. The Land Within: Indigenous Territory and The Perception of the Environment. IWGIA-International Work Group for Indigenous Affairs, Copenhagen.

Godoy, M.

2017 Las rutas del Sollipulli hacia el Puel Mapu. Revista Austral de Ciencias Sociales, 27: 45-69.

González-Parra, C., y Simon, J.

2008 All that glitters is not gold: Resettlement, vulnerability, and social exclusion in the Pehuenche community Ayin Mapu, Chile. American behavioral scientist, 51: 1774-1789.

Gordillo, G.

2004 Landscapes of devils: Tensions of place and memory in the Argentinean Chaco. Duke University Press, Princeton.

Gundermann, H.; González, H. y De Ruyt, L.

2009a. Migración y movilidad mapuche a la Patagonia Argentina. Magallania, 37: 21-35.

Gundermann, H.; González, H. y De Ruyt, L.

2009b. Migración y movilidad mapuche entre Araucanía y Nor Patagonia. Avá, 15: 103-123.

Huiliñir-Curío, V.

2015 Los senderos pehuenches en Alto Biobío (Chile): articulación espacial, movilidad y territorialidad. Revista de Geografía Norte Grande, 62: 47-66.
Huiliñir-Curío, V.

2018 De senderos a paisajes: paisajes de las movilidades de una comunidad mapuche en los Andes del sur de Chile. Chungara, 50, 3: 487-499.

Imilan, W.; Jirón, P. e Iturra, L.

2015 Más allá del barrio: habitar Santiago en la movilidad cotidiana. Antropologías del Sur, 3: 87-103.

Iturra, L. y Jirón, P.

2016 Siguiendo trayectorias, dibujando trayectos. Construcción de diagramas desde la experiencia de los habitantes. AUS, 1 (19): 4-9.

Ingold, T.

2011 Being Alive: Essays on Movement, Knowledge and Description. Routledge, London.

Ingold, T.

2012 Ambientes para la vida. Ediciones Trilce, Montevideo.

Jirón, P.

2011 On becoming la sombra/the shadow. En: Buscher, Monika, Urry, John, Witchger, Katian (eds). 2011. Mobile Methods. Routledge, London.

Jirón, P.; Lange, C. y Bertrand S., M.

2010 Exclusión y desigualdad espacial: retrato desde la movilidad cotidiana. INVI, 25 (68).

Jouffe, Y. y Lazo, A.

2010 Las prácticas cotidianas frente a los dispositivos de la movilidad: Aproximación política a la movilidad cotidiana de las poblaciones pobres periurbanas de Santiago de Chile. EURE, 36 (108): 9-47.

Lazo, A. y Carvajal, D.

2018 La movilidad y el habitar chilote. Cambios, rupturas y continuidades en las prácticas de movilidad cotidiana de los habitantes del archipiélago de Chiloé, en el sur austral de Chile. Chungara, 50, 1: 145-154.

Lazo, A. y Carvajal, D.

2017 Habitando la movilidad: El viaje en lancha, los objetos y la experiencia de la movilidad en el archipiélago de Quinchao, Chiloé. Austral de Ciencias Sociales, 33: 89-102.

Legg, $\mathrm{S}$.

2005 Contesting and surviving memory: space, nation, and nostalgia in Les Lieux de Mémoire. Environment and Planning D: Society and Space, 23: 481-504.

Lindón, A.

1999 Narrativas autobiográficas, memoria y mitos: una aproximación a la acción social. Economía, sociedad y territorio, 2 (6).

Mendoza, J.

2005 La forma narrativa de la memoria colectiva. Polis, 1 (1): 9-30.

Milbourne, P., \& Kitchen, L.

2014 Rural mobilities: Connecting movement and fixity in rural places. Journal of Rural Studies, 34: 326-336.

Norman, M. E., Petherick, L., Garcia, E., Glazebrook, C., Giesbrecht, G., y Duhamel, T.

2015 Examining the more-than-built environments of a northern Manitoban community: Re-conceptualizing rural indigenous mobilities. Journal of Rural Studies, 42, 166-178. 
Panelli, R., Hubbard, P., Coombes, B., y Suchet-Pearson, S. 2009 De-centring White ruralities: ethnicity and indigeneity. Journal of Rural Studies, 25 (4): 355-443.

Quintriqueo, M.; Quilaqueo, R. y Torres, H.

2014 Contribución para la enseñanza de las ciencias naturales: saber mapuche y escolar. Educação e Pesquisa, 40 (4): 965-982.

Ramos, A.

2014 Cuando la memoria es un proyecto de restauración: el potencial relacional y oposicional de conectar experiencias. En Historias y memorias. Diálogos desde una perspectiva interdisciplinaria, editado por Bello, A; González, Y.; Rubilar, P. y Ruiz, O. (Eds.), Temuco: Ediciones UFRO, Temuco.

Ramos, R

2018 Entre poder y resistencias: Análisis de las movilidades territoriales en la zona fronteriza de la región de Tarapacá, Chile. Diálogo Andino (57): 87-98.

Riquelme, H. y Riquelme, M.

2018. Representando el espacio: experiencias de movilidad cotidiana a partir de la confección de mapas en La Araucanía. Estudios Socioterritoriales, 23: 101-117.
Riquelme, H. y Lazo, A.

2019 Trazando rutinas: el papel del ferrocarril en las prácticas cotidianas y movilidades interurbanas de habitantes de La Araucanía, Chile. Urbe. Revista Brasileira de Gestão Urbana, 11: 1-15.

Salazar, G.; Fonck, M. e Irarrázaval, F.

2017 Paisajes en movimiento: sentidos de lugar y prácticas interculturales en ciudades de la región de La Araucanía, Chile. Chungara, 49 (2): 251-264.

Sletto, B.

2016 Indigenous mobilities, territorialization, and dispossession in the Sierra de Perijá, Venezuela: Rescuing lands and meanings in Hábitat Indígena Yukpa, Toromo-Tütari. Geoforum, 74: 117-127.

Vidal, E.; Calfuqueo, J. y Ancan, L.

2017 Espacio y tiempo en la construcción de la dimensión de aprendizaje rakizuam ka mapuzugun, a partir del saber de algunos kimce del bafkeh mapu. En Becerra, R. \& Llanquinao G. (editores). Mapun kimün: Relaciones mapunche entre persona, tiempo y espacio. Ocho Libros Editores, Santiago. 
